\title{
Resiliência, depressão e qualidade de vida de pacientes com câncer de cabeça e pescoço no pré-operatório
}

\author{
Resilience, depression and quality of life of patients with head and neck cancer in the \\ preoperative period
}

Resiliencia, depresión y calidad de vida de pacientes con cáncer de cabeza y cuello en el
período preoperatorio

Ywia Danieli Valadares ${ }^{1 *}$, João Marcos Arantes Soares ${ }^{1}$, Ana Paula Madureira², Michele Conceição Pereira1.

\section{RESUMO}

Objetivo: Avaliar os escores de resiliência, qualidade de vida e depressão em pacientes com Carcinoma de Células Escamosas de Cabeça e Pescoço (CCECP) no pré-operatório e correlacionar tais variáveis. Métodos: $\mathrm{Na}$ fase pré-operatória do tratamento cirúrgico, analisaram-se 28 pacientes com CCECP diagnosticados em um hospital do centro-oeste de Minas Gerais, entre agosto de 2017 e novembro de 2018. Foi utilizado o Questionário de Qualidade de Vida (QV) da Universidade de Washington, o Inventário de Depressão de Beck e a Escala de Resiliência. Resultados: Encontrou-se uma correlação baixa e negativa entre a resiliência e a depressão $(r=-0,497 ; p=0,007)$, uma alta e negativa $(r=-0,708 ; p=0,000)$ entre a depressão e a QV e uma moderada e positiva entre a resiliência e a $Q V(r=0,512 ; p=0,005)$. Conclusão: A resiliência interferiu positivamente na depressão e na QV dos pacientes estudados. Em contraste, a QV se correlacionou negativamente com a depressão. Assim, a resiliência representou uma importante variável, que deveria ser introduzida no processo de triagem dos pacientes com CCECP, já que pode contribuir para uma melhora da QV e depressão.

Palavras-chave: Neoplasias de cabeça e pescoço, Carcinoma de células escamosas, Resiliência psicológica, Qualidade de vida, Depressão.

\begin{abstract}
Objective: To evaluate the scores of resilience, quality of life and depression in patients with Head and Neck Squamous Cells Carcinoma (HNSCC) in the preoperative period and correlate these variables. Methods: In the preoperative phase of surgical treatment, 28 patients diagnosed with HNSCC diagnosed in a hospital in the midwest of Minas Gerais between August 2017 and November 2018 were analyzed. The Quality of Life Questionnaire (QOL) from the University of Washington, the Beck Depression Inventory and the Resilience Scale was used in this study. Results: A low and negative correlation was found between resilience and depression $(r=-0,497 ; p=0.007)$, a high and negative correlation $(r=-0.708 ; p=0.000)$ between depression and $\mathrm{QOL}$ and a moderate and positive between resilience and QOL $(r=0.512 ; p=0.005)$. Conclusion: Resilience positively interfered in the depression and QOL of the studied patients. In contrast, QL was negatively correlated with depression. Thus, resilience represented an important variable, which should be introduced in the screening process for patients with HNSCC, since it can contribute to an improvement in QOL and depression.
\end{abstract}

Keywords: Head and neck neoplasms, Squamous cell carcinoma, Psychological resilience, Quality of life, Depression.

\section{RESUMEN}

Objetivo: Evaluar los puntajes de resiliencia, calidad de vida y depresión en pacientes con Carcinoma Epidermoide de Cabeza y Cuello (CECC) en el período preoperatorio y correlacionar estas variables. Métodos: En la fase preoperatoria del tratamiento quirúrgico se analizaron 28 pacientes diagnosticados de

1 Universidade Federal de São João Del Rei (UFSJ), Divinópolis - MG. *E-mail: ywiaval@hotmail.com

2 Universidade Federal de São João Del Rei (UFSJ), São João Del Rei - MG. 
CECC diagnosticados en un hospital del medio oeste de Minas Gerais entre agosto de 2017 y noviembre de 2018. Se utilizó el Cuestionario de Calidad de Vida (CV) de la Universidad de Washington, el Inventario de Depresión de Beck y la Escala de Resiliencia. Resultados: Se encontró una correlación baja y negativa entre resiliencia y depresión $(r=-0,497 ; p=0.007)$, una correlación alta y negativa $(r=-0.708 ; p=0.000)$ entre depresión y CV y una correlación moderada y positiva. entre resiliencia y CV $(r=0,512 ; p=0,005)$. Conclusión: la resiliencia interfirió positivamente en la depresión y la calidad de vida de los pacientes estudiados. Por el contrario, la calidad de vida se correlacionó negativamente con la depresión. Así, la resiliencia representó una variable importante, que debe introducirse en el proceso de cribado de los pacientes con CECC, ya que puede contribuir a una mejora de la CV y la depresión.

Palabras clave: Neoplasias de cabeza y cuello, Carcinoma de células escamosas, Resiliencia psicológica, Calidad de vida, Depresión.

\section{INTRODUÇÃO}

Emprega-se o termo Câncer de Cabeça e Pescoço (CCP) para se referir a um grupo heterogêneo de tumores malignos que se desenvolvem na mucosa do trato aerodigestivo superior, englobando a cavidade oral, lábio, faringe e laringe (GOMES-FERRAZ C, et al., 2019).

Grande parte dessas neoplasias malignas atinge as células escamosas que revestem as superfícies da mucosa do aparelho digestivo superior. Elas são classificadas de acordo com a área da Cabeça e Pescoço (CP) em que se iniciam e referidas como Carcinomas de Células Escamosas da Cabeça e Pescoço (CCECP), já que este é o tipo histológico mais comum, presente em mais de 90\% dos casos (SIEGEL RL, et al., 2019).

No Brasil, para o triênio 2020-2022, estimam-se 22.840 novos casos de câncer de cavidade oral e laringe (15.190 em homens e 7.650 em mulheres), sendo que, entre os homens, o câncer de cavidade oral ocupa a quinta posição e o de laringe a oitava (INSTITUTO NACIONAL DO CÂNCER JOSÉ ALENCAR GOMES DA SILVA (INCA), 2019).

A etiologia do CCP é multifatorial, contudo, o tabaco e o consumo excessivo de álcool compreendem os fatores de risco mais conhecidos (GONG SQ, et al., 2019). Salienta-se que para os tumores de lábio, a exposição crônica à radiação ultravioleta apresenta grande importância, assim como a infecção pelo papilomavírus humano (HPV) para os tumores orofaríngeos (DEVINS KM, et al., 2019).

O diagnóstico precoce do CCECP na maior parte dos casos é difícil e tardio. Isso deve-se provavelmente a três razões: demora do paciente em procurar assistência, desqualificação dos profissionais de saúde para diagnóstico/seguimento e poucos serviços de referência habilitados para o tratamento (HUANG C, et al., 2019).

A localização do tumor e o estágio da doença influenciam diretamente no aparecimento dos sintomas. Para os tumores de cavidade oral em fase inicial são observados dor, ulcerações de difícil cicatrização, manchas vermelhas ou brancas, dificuldade de engolir e possíveis alterações na dentição. Os tumores de orofaringe, hipofaringe e laringe apresentam sintomas como dor de garganta, rouquidão e otalgia em fases mais avançadas. Por esse motivo, são frequentemente diagnosticados em estágios avançados (ROCHA BQC, et al., 2017).

O sistema de estadiamento TNM é usado mundialmente para a classificação de tumores malignos desde 1943. Em 1950 a União Internacional Contra o Câncer (UICC), o adotou como base para a classificação de estádios clínicos sugeridos pela OMS. Tem como objetivo avaliar o grau de disseminação do tumor, usando os atributos Tumor (T), Linfonodo (N) e Metástase (M). Nesse sistema de classificação dos tumores malignos, T se refere a extensão anatômica do tumor primário, $\mathrm{N}$ a ausência ou presença e extensão de metástases em linfonodos regionais e $M$ a presença ou ausência de metástases a distância, recebendo graduação de T0 a T4, N0 a N3 e M0 a M1 (HUANG SH e O'SULLIVAN B, 2017).

A escolha do tratamento se baseia na localização do tumor, estádio clínico, expectativas e condições clínicas e físicas do paciente, bem como na ressecabilidade do tumor e viabilidade de preservação dos órgãos (HUANG C, et al., 2019). 
Nos estágios iniciais da doença o tratamento é unimodal, com uso de cirurgia ou radioterapia, o que dependerá da localização do tumor e das condições clínicas do paciente. Nos estágios avançados o tratamento faz-se com associação: cirurgia associado a radioterapia pós-operatória, associada ou não a quimioterapia, o que confere melhor controle locorregional e sobrevida, mas resulta em diversos efeitos colaterais físicos, funcionais e emocionais, que podem repercutir na vida diária do paciente e de sua família, uma vez que a ideia de finitude da vida se torna presente (MELO MCB, et al., 2012; ROCHA BQC, et al.,2017).

Alguns estudos têm demonstrado que pacientes com CCP podem apresentar distúrbios psiquiátricos e/ou emocionais (BOYES AW, et al., 2013; SAWADA NO, et al., 2012). A depressão é a variável psicológica mais estudada em relação à progressão da doença e mortalidade, pois, pacientes deprimidos podem ter o risco de mortalidade, 26 a 39\% maior que seus pares não deprimidos (ZIMMARO LA, et al., 2018). Resultados semelhantes foram apresentados por Kim S, et al. (2016), que demostraram uma correlação entre a depressão e o risco de mortalidade precoce em pacientes com CCP e sugerem que a depressão é um fator prognóstico de sobrevida em dois a três anos.

Os sintomas, tratamentos e consequências dos CCECP avançados, podem causar impacto devastador na qualidade de vida $(\mathrm{QV})$ dos pacientes. Muitos adquirem deficiências na alimentação, fala e respiração, associadas a desfiguração facial (ALGTEWI E, et al., 2017). Por isso, vários trabalhos investigaram a QV, depressão e suas correlações em pacientes com CCP (DE GRAEFF A, et al., 2000; GOMES-FERRAZ C, et al., 2019; ZIMMARO LA, et al., 2018).

Diante do sofrimento causado pelo CCECP e seu tratamento, alguns pacientes conseguem superar as dificuldades e utilizam dessas como uma forma de crescimento pessoal, tornando-se mais resilientes (LIMA RJ, et al., 2020).

O estudo da resiliência ganhou força nas últimas décadas, principalmente pelos profissionais da psicologia, e seu uso em pesquisas com pacientes oncológicos tem crescido (HU et al., 2018; SHARPLEY CF, et al., 2014).

O termo resiliência se refere à capacidade que alguns indivíduos apresentam de superar situações adversas graves e conseguir transformá-las em vantagens ou estímulo para o seu desenvolvimento biopsicossocial e também pode ser conceituada como a habilidade dos pacientes em enfrentar e encarar eventos traumáticos, como um tratamento de câncer, sendo um parâmetro de capacidade do paciente em manter suas funções físicas, emocionais e psicológicas (WAGNILD G, 2009).

$O$ estudo da resiliência em pacientes com CCECP ainda é carente. Em um estudo retrospectivo, Clarke G, et al. (2019), avaliaram a associação entre resiliência e QV em pacientes com CCP, porém, não consideraram a variável depressão. Sendo assim, trabalhos com esse foco poderão auxiliar no planejamento de intervenções visando reduzir os impactos psicológicos da doença e da intervenção e melhorar a reabilitação e adesão ao tratamento.

Nesse contexto, o presente estudo teve como objetivo avaliar a resiliência, depressão e QV em pacientes com CCECP no período pré-operatório.

\section{MÉTODOS}

O presente estudo foi aprovado pelo Comitê de Ética em Pesquisa Envolvendo Seres Humanos do Campus Centro-Oeste Dona Lindu, da Universidade Federal de São João Del Rei (protocolo no 2.172.356) e pelo Comitê de Ética em Pesquisa do Hospital São João de Deus (protocolo no 2.225.907). O consentimento livre e esclarecido foi assinado por todos os voluntários.

Os participantes do estudo foram recrutados na Clínica de cirurgia de cabeça e pescoço da Unidade Oncológica de um hospital de referência do interior de Minas Gerais, Brasil. Selecionaram-se pacientes com diagnóstico de CCECP entre agosto de 2017 e novembro de 2018.

Os critérios de inclusão foram: (I) pacientes com tumor primário diagnosticado como CCE localizado na boca, faringe, hipofaringe e laringe, confirmado por biópsia; (II) pacientes submetidos à cirurgia como 
tratamento inicial com finalidade curativa e (III) pacientes com ausência de alterações cognitivas identificadas pelo Mini Exame do Estado Mental (BERTOLUCCI PHF, et al., 1994). Os critérios de exclusão compreenderam: (I) presença de outros tumores primários simultâneos; (II) pacientes com metástase à distância na época da admissão e (III) ausência de realização de alguma das avaliações do protocolo.

Após a avaliação dos cirurgiões e indicação do procedimento cirúrgico, cada voluntário respondeu a uma ficha de identificação contendo dados demográficos, história clínica, fatores de risco e exame locorregional. Também foi aplicado o teste Mini Exame do Estado Mental (MEEM) para avaliação cognitiva, composto por sete categorias que mensuram funções cognitivas específicas: orientação para tempo e local, registro de três palavras, atenção e cálculo, lembrança de três palavras, linguagem e capacidade construtiva visual (BERTOLUCCI PHF et al., 1994).

O escore varia de zero a 30 pontos, sendo o ponto de corte para alteração cognitiva: 20 pontos para analfabetos, 25 pontos para idosos com um a quatro anos de estudo, 26,5 pontos para idosos com cinco a oito anos de estudo, 28 pontos para aqueles com nove a 11 anos de estudo e 29 pontos para aqueles com mais de 11 anos de estudo (BRUCKI SMD, et al., 2003).

Ainda na avaliação, os voluntários responderam ao Questionário de Avaliação de Qualidade de Vida da Universidade de Washington (UW-QOL) - $4^{\text {a }}$ versão, que consta de 12 domínios que avaliam dor, aparência, atividade, recreação, deglutição, mastigação, fala, ombro, paladar, saliva, humor e ansiedade, sendo que, cada questão permite descrever as disfunções ou limitações vividas diariamente pelo paciente, possuindo entre três e cinco possibilidades de escolha, resultando numa escala de zero (pior) a 100 pontos (melhor) QV (VARTANIAN JG, et al., 2006).

Para avaliar a depressão utilizou-se o Inventário de Depressão de Beck, composto por 21 questões objetivas variando de zero a 3 pontos. Os itens descrevem a tristeza, pessimismo, sensação de fracasso, falta de satisfação, sensação de culpa, sensação de punição, auto depreciação, autoacusações, ideias suicidas, crises de choro, irritabilidade, retração social, indecisão, distorção da imagem corporal, inibição para 0 trabalho, distúrbio do sono, fadiga, perda de apetite, perda de peso, preocupação somática, diminuição de libido, numa pontuação que varia de 0 a 63 . Escores de 0 a 11: indicam pacientes sem depressão ou depressão mínima; escores de 12 a 19: depressão leve; escores de 20 a 35: depressão moderada e escores de 36 a 63: depressão grave (GORESTEIN C e ANDRADE L, 1998).

Para finalizar a coleta foi aplicada a Escala de Resiliência proposta por Wagnild G e Young H (1993), com 25 itens avaliados por uma escala de Likert, variando de um (discordo totalmente) a sete pontos (concordo totalmente). O escore total varia de 25 a 175 pontos, categorizado em baixo (menor ou igual a 124 pontos); médio (125 a 145 pontos) e alto (maior que 145 pontos) (WAGNILD G, 2009).

Os dados foram analisados pelo software Statistic Package for Social Sciences (SPSS) for Windows, versão 23.0 (SPSS Inc., Estados Unidos). O Teste de Kolmogorov-Smirnov avaliou a normalidade dos dados e o teste de correlação de Pearson foi usado para correlacionar as variáveis. O valor de alfa foi estabelecido em 0.05 , correspondendo a um intervalo de confiança de $95 \%$.

\section{RESULTADOS E DISCUSSÃO}

Incialmente, a amostra foi constituída por 34 pacientes, entretanto, seis foram excluídos por não apresentarem o cognitivo preservado segundo o MEEM. A idade mínima dos pacientes foi de 37 anos e a máxima de 88 anos, com média de $57,07 \pm 10,37$ anos. A maioria dos pacientes pertencia ao gênero masculino $(89,30 \%)$, quinze pacientes $(53,57 \%)$ apresentavam antecedentes familiares para a doença e a maior parte era tabagista $(85,71 \%)$ e etilista $(64,28 \%)$.

As características demográficas descritas acima, bem como a exposição aos fatores de risco e história familiar, estão de acordo com os relatos da literatura. Gomes-Ferraz C, et al. (2019) e Kfouri AS, et al. (2018), avaliaram a mesma população e também confirmaram a maior prevalência na quinta e sexta décadas de vida e maior incidência em tabagistas e/ou etilistas e com história familiar positiva. 
No exame locorregional, a maior parte dos pacientes tinha tumores localizados na cavidade oral $(50 \%)$, seguido por laringe $(28,57 \%)$ e faringe $(21,43 \%)$, dados que corroboram com os resultados de outros estudos (DE MELO NB, et al., 2019; HUANG C, et al., 2019). E estavam em estágios T4 e N0, também observados em alguns estudos epidemiológicos com a mesma população (Tabela 1) (BRADSHAW PT, et al., 2012; CHAVONI RC, et al., 2014).

Tabela 1 - Distribuição de frequência dos entrevistados com carcinoma de células escamosas de cabeça e pescoço, segundo o exame locorregional e estadiamento.

\begin{tabular}{lcc}
\hline Características & $\mathbf{n}$ & $\%$ \\
\hline Local do tumor & 5 & 17,86 \\
\hline Língua & 4 & 14,29 \\
Lábio & 3 & 10,71 \\
Assoalho de boca & 2 & 7,14 \\
Mucosa Jugal & 4 & 4,29 \\
Hipofaringe & 2 & 7,14 \\
Orofaringe & 8 & 28,57 \\
Laringe & & \\
\hline Estágio T & 8 & 28,57 \\
\hline T1 & 4 & 14,29 \\
T2 & 6 & 21,42 \\
T3 & 10 & 35,71 \\
T4 & & \\
\hline Estádio N & 16 & 57,14 \\
\hline N0 & 3 & 10,71 \\
N1 & 5 & 17,86 \\
N1 & 4 & 14,29 \\
N3 & & \\
\hline Estadiamento do Tumor & 6 & 21,42 \\
\hline Estádio I & 3 & 10,71 \\
Estádio II & 5 & 17,86 \\
Estádio III & 14 & 50 \\
Estádio IV & $\mathbf{2 8}$ & $\mathbf{1 0 0}$ \\
\hline TOTAL &
\end{tabular}

Legenda: $n$ : Frequência absoluta; \%: Frequência relativa; T: Tumor; N: Linfonodo.

Fonte: Valadares YD, et al., 2021.

$\mathrm{Na}$ análise da pontuação total da resiliência, a maioria dos pacientes se declarou com alta resiliência e sem depressão (Tabela 2).

A alta resiliência pode ser atribuída ao fato de serem a maior parte idosos e que devida as suas experiências passadas, dão um sentido diferente para as dificuldades, avaliando positivamente os desafios impostos e considerando novos caminhos para a vida (LIMA RJ, et al., 2020). Resultados semelhantes foram observados em outros estudos com população oncológica (DIMITROVSKA GR, et al., 2015; HU T, et al., 2018).

No que diz respeito a depressão, grande parte dos pacientes apresentou-se sem sintomas de depressão, o que contraria estudos anteriores que demonstram ser a depressão uma desordem frequente em pacientes com CCP, associada ao comprometimento funcional, como alterações na fala, deglutição, respiração, paladar e desconfiguração facial que ocorrem durante o tratamento, comprometendo a QV dos pacientes (KIM S, et al., 2016; ROHDE RL, et al., 2018).

Alguns fatores podem ter contribuído para esse resultado da depressão, entre eles o fato dos pacientes estarem no início do tratamento e não terem sofrido alterações na imagem corporal. Quando questionados sobre a mudança na aparência após o diagnóstico de câncer, a maioria relatou mudanças mínimas ou nenhuma. Rhoten BA, et al. (2014), avaliaram a depressão em pacientes com CCP e relatam que as mudanças de aparência física são as principais preditoras da depressão nos pacientes. 
Outro aspecto que pode ter contribuído para os baixos escores de depressão encontrados é o predomínio do gênero masculino (89,3\%). A depressão é apontada, mais frequentemente, na população feminina, indicando que as mulheres são mais propensas aos transtornos depressivos ou expressam mais os sintomas para os entrevistadores (LEE SJ e CARTMELL KB, 2020).

Tabela 2 - Níveis de resiliência e depressão nos entrevistados.

\begin{tabular}{lcc}
\hline Variáveis & $\mathbf{n}$ & $\%$ \\
\hline Resiliência & & \\
\hline Alta resiliência & 16 & 57,14 \\
Moderada resiliência & 7 & 25 \\
Baixa resiliência & 5 & 17,86 \\
\hline Depressão & & \\
\hline Sem depressão & 15 & 53,57 \\
Depressão leve & 8 & 28,57 \\
Depressão moderada & 3 & 10,71 \\
Depressão grave & 2 & 7,14 \\
\hline
\end{tabular}

Legenda: $\mathrm{n}$ : Frequência absoluta, \%: Frequência relativa.

Fonte: Valadares YD, et al., 2021.

Dos doze domínios do Questionário de Avaliação de Qualidade de Vida da Universidade de Washington (UW-QOL) avaliados, o pior escore foi a ansiedade com média de 33 pontos (Gráfico 1).

Pacientes com CCP experienciam altos níveis de ansiedade em comparação com outras populações oncológicas, explicadas por uma combinação de fragilidades preexistentes, altos níveis de sintomas da doença e déficits funcionais, aumentando as chances desse distúrbio (HENRY M, et al., 2018).

Suzuki M, et al. (2016) observaram que grande parte dos pacientes com CCP avaliados tinham altos níveis de ansiedade no pré-operatório, o mesmo também foi relatado por Hortense FTP, et al. (2020) que mostraram ser o período entre o diagnóstico e a decisão terapêutica um momento propício para o desenvolvimento de ansiedade, em consequência da preocupação com uma possível cirurgia, com o próprio tratamento e incerteza quanto à recuperação.

Gráfico 1 - Distribuição dos domínios referentes a qualidade de vida.

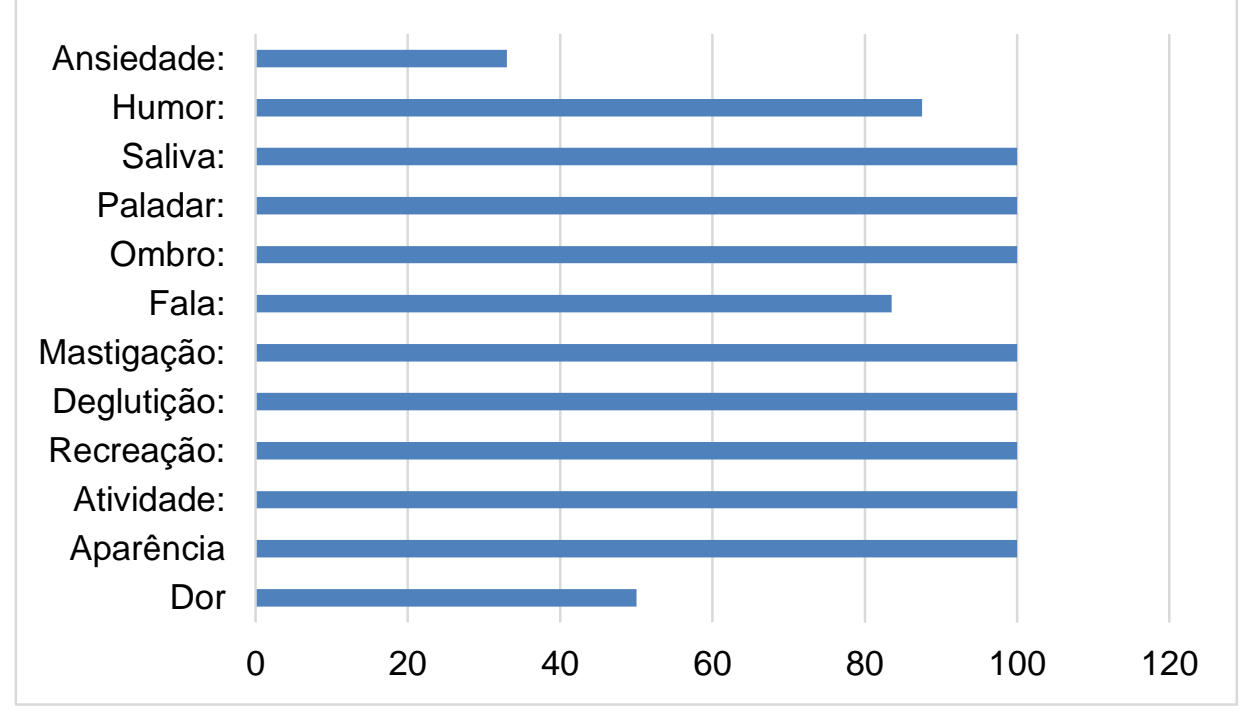

Fonte: Valadares YD, et al., 2021.

Para as análises de correlações, utilizou-se a soma dos escores totais das variáveis e encontrou-se uma correlação baixa e negativa entre a resiliência e depressão, mostrando que quando a resiliência do paciente aumenta, a depressão reduz, sendo esse estudo pioneiro nessa investigação para o CCP (Gráfico 2). 
Gráfico 2 - Correlação das variáveis: resiliência e depressão.

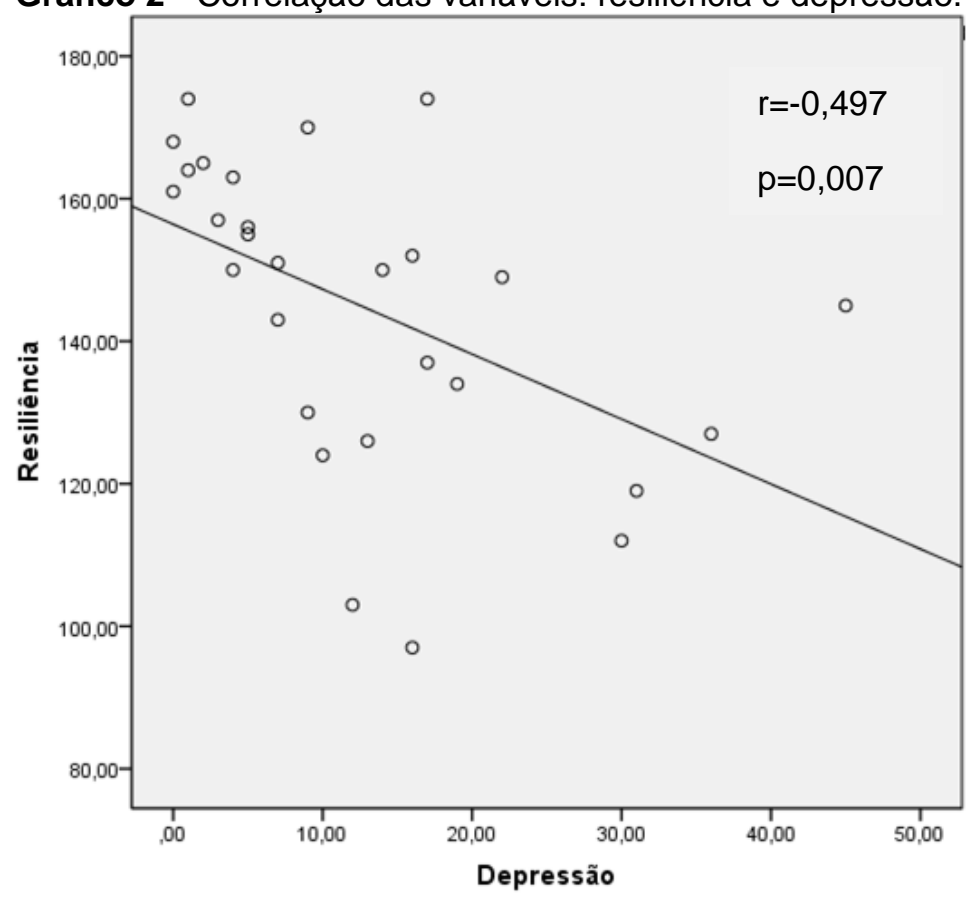

Fonte: Valadares YD, et al., 2021.

Essa correlação nos atenta que nem sempre o diagnóstico e o tratamento do câncer causam apenas efeitos negativos aos pacientes e, há alguns anos, pesquisas evidenciam os efeitos positivos do câncer nos sobreviventes. Estes trabalhos mostram aspectos da experiência que os pacientes veem como positivos, tais como: reavaliação dos valores, conscientização sobre a importância da saúde física e emocional, melhorias nas relações pessoais, familiares e mudanças nas prioridades da vida (LUSZCZYNSKA A, et al., 2005; THAMBYRAJAH C, et al., 2010).

Poucos estudos abordam a correlação entre resiliência e depressão em pacientes oncológicos. Sharpley CF, et al. (2014) avaliaram essas variáveis em pacientes com câncer de próstata e observaram uma correlação inversa entre ambas, afirmando que um relatório focado nos aspectos específicos da resiliência pode ser de potencial assistência a esses pacientes na resistência à depressão.

Dimitrovska GR, et al. (2015), avaliaram a correlação entre resiliência e depressão em indivíduos com câncer de mama. Os resultados mostraram que pacientes menos deprimidos tinham níveis mais altos de resiliência, sugerindo que a resiliência pode contribuir para níveis mais baixos de depressão.

Hu T, et al. (2018), avaliaram 289 pacientes com câncer de pulmão quanto à resiliência e depressão. As análises de correlações revelaram que a depressão estava associada negativamente à resiliência, o que corrobora com os resultados do presente trabalho.

$\mathrm{Na}$ análise de correlação da qualidade de vida e depressão, observou-se uma correlação alta e negativa entre a QV e depressão (Gráfico 3).

Duffy AS, et al. (2002) relataram que a avaliação da QV tem sido cada vez mais importante nos serviços de saúde, particularmente na área da oncologia, onde ter QV é tão importante quanto ter quantidade de vida. Os mesmos autores conduziram um estudo piloto que avaliou os efeitos do tabagismo, álcool e depressão em 81 indivíduos com CCP. Entre todos os preditores os sintomas depressivos tiveram associação mais forte com a QV, sugerindo que se trata de um problema grave, geralmente, não abordado e investigado. Os autores sugerem que isso esteja relacionado ao fato de que a depressão não é prioridade para os profissionais de saúde, que concentram suas preocupações nas questões fisiológicas do CCP.

Um estudo conduzido em um Hospital de Ribeirão Preto avaliou 41 pacientes com CCP submetidos à radioterapia em três diferentes momentos: início, quinze dias após o início do tratamento e no final do 
tratamento. Observou-se que os escores de depressão aumentaram e a QV reduziu durante o decorrer do tratamento, sendo ambos os sintomas correlacionados. Observaram ainda que a presença de depressão influenciou negativamente a QV (SAWADA NO, et al., 2012).

Gráfico 3 - Correlação das variáveis: Qualidade de vida e depressão.

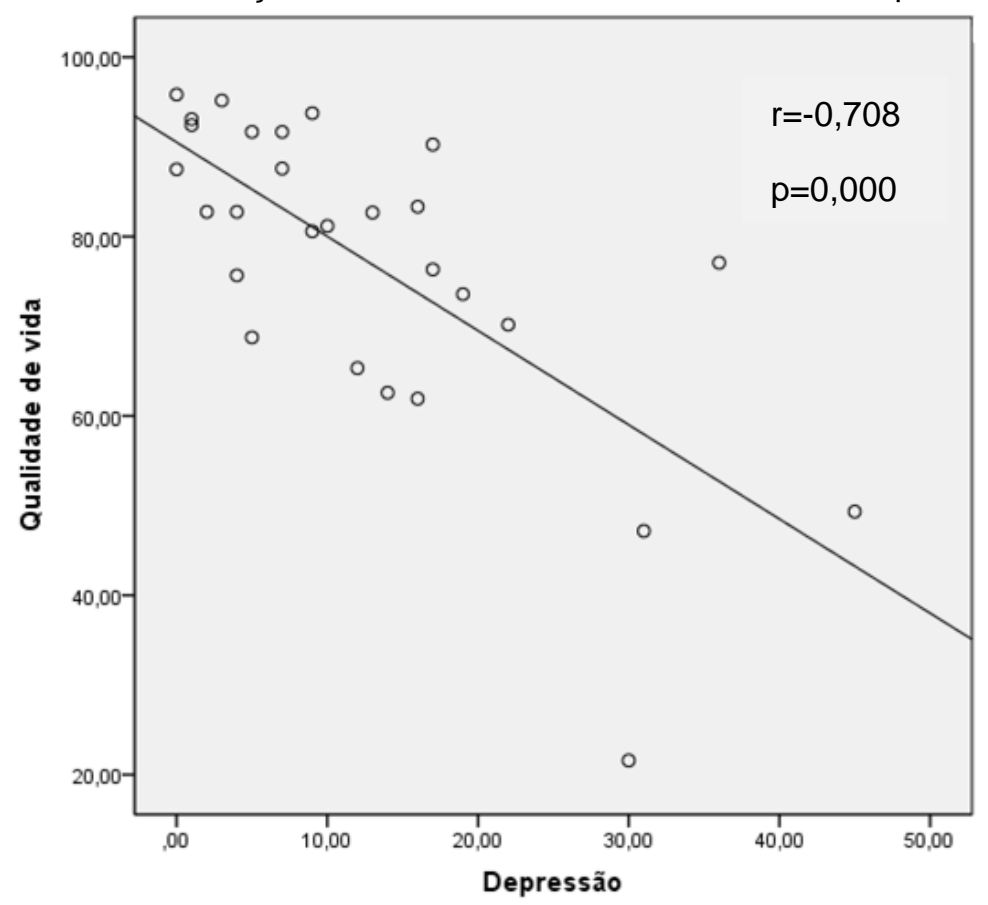

Fonte: Valadares YD, et al., 2021.

Quando correlacionado a resiliência e a QV, encontrou-se uma correlação moderada e positiva entre a resiliência e QV (Gráfico 4).

Gráfico 4 - Correlação das variáveis: Resiliência e qualidade de vida.

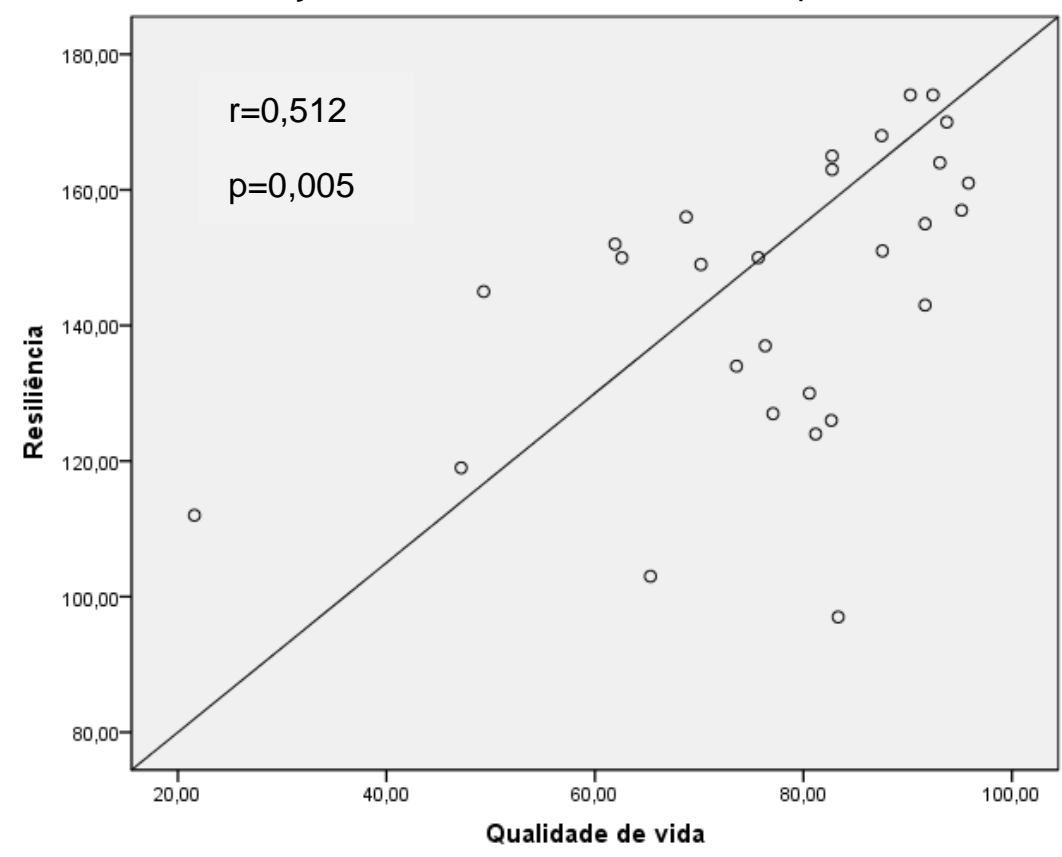

Fonte: Valadares YD, et al., 2021. 
Recentemente, Clarke G, et al. (2019), avaliaram a resiliência e QV em pacientes com CCP em tratamento, empregando o questionário de qualidade de vida UW-QOL e a escala de resiliência de Connor-Davidson. Os autores observaram que um aumento na resiliência previa um pequeno aumento na QV, corroborando com os dados encontrados no presente estudo. $O$ valor mais robusto encontrado no nosso estudo em comparação com o obtido pelos autores do trabalho supracitado $(R=0,512$ e $p=0,005)$, se deve, possivelmente, ao fato de que $o$ presente trabalho entrevistou apenas pacientes com um determinado tipo histológico de tumor submetidos à tratamento cirúrgico. Além disso, todos os pacientes que tiveram tumores prévios foram excluídos.

Esse estudo apresenta algumas limitações, tais como: tamanho reduzido da amostra, considerando que foram incluídos somente pacientes com CCECP submetidos a tratamento cirúrgico e com cognitivo preservado. Entretanto, é um trabalho pioneiro na investigação da qualidade de vida, resiliência e depressão em pacientes com CCECP. Reforça-se a necessidade da realização de trabalhos futuros avaliando a importância da triagem da resiliência e da depressão no momento do diagnóstico, para melhor reabilitação do paciente.

\section{CONCLUSÃO}

A análise dos dados sugere um maior comprometimento do domínio ansiedade em consequência do diagnóstico do CCECP. O estudo apontou correlações entre a resiliência e QV, resiliência e depressão e depressão e QV. Sendo assim, torna-se necessária a capacitação de todos os profissionais envolvidos no tratamento desses pacientes quanto a importância dessa investigação, sendo que essa estratégia contribuirá para importantes decisões no tratamento, como a seleção dos indivíduos que podem se beneficiar de intervenções cirúrgicas precoces, trabalhar formas de reduzir os níveis de depressão e aumentar a resiliência, levando a um aumento da QV dos pacientes e sucesso do tratamento.

\section{REFERÊNCIAS}

1. ALGTEWI E, et al. Online support groups for head and neck cancer and health-related quality of life. Quality of Life Research, 2017; 26(9): 2351-2362.

2. BERTOLUCCI PHF, et al. O mini exame do estado mental em uma população geral- Impacto da escolaridade. Arquivos de neuropsiquiatria, 1994; 52(1): 1-7.

3. BOYES AW, et al. Prevalence and predictors of the short-term trajectory of anxiety and depression in the first year after a cancer diagnosis: A population-based longitudinal study. Journal of Clinical Oncology, 2013; 31(21): 2724-2729.

4. BRADSHAW PT, et al. Associations Between Dietary Patterns and Head and Neck Cancer. American journal of epidemiology, 2012; 175(12): 1225-1233.

5. BRUCKI SMD, et al. Sugestões para o uso do mini-exame do estado mental no Brasil. Arquivos de Neuro-Psiquiatria, 2003; 61(3): 777-781.

6. CHAVONI RC, et al. Diagnóstico nutricional de pacientes do serviço de cabeça e pescoço e sua relação com a disfagia em um hospital oncológico do Paraná. Revista Brasileira Cirurgia Cabeça Pescoço, 2014; 43(1): 35-41.

7. CLARKE G, et al. Exploring the relation between patients' resilience and quality of life after treatment for cancer of the head and neck. British Journal of Oral and Maxillofacial Surgery, 2019: 5-9.

8. DE GRAEFF A, et al. Pretreatment Factors predicting quality of life treatment for head and neck cancer. Head \& Neck, 2000; 22: 398-407.

9. DE MELO NB, et al. Oral health related quality of life and determinant factors in patients with head and neck cancer. Medicina Oral Patologia Oral e Cirugia Bucal, 2019; 24(3): 281-289.

10. DEVINS KM, et al. The evolving landscape of HPV-related neoplasia in the head and neck. Human Pathology, 2019; 94: 29-39.

11. DIMITROVSKA GR, et al. Depression and Resilience in Breast Cancer Patients. Journal of Medical Sciences, 2015; 3(4): 661-665.

12. DUFFY SA, et al. Effect of smoking, alcohol, and depression on the quality of life of head and neck cancer patients. General Hospital Psychiatry, 2002; 24: 140-147.

13. GOMES-FERRAZ C, et al. Uso de tecnologia de comunicação alternativa na avaliação da qualidade de vida de pacientes com câncer de cabeça e pescoço. Cadernos Brasileiros de Terapia Ocupacional, 2019; 27(1): 61-71. 
14. GONG SQ, et al. The Expression and Effection of MicroRNA-499a in High-Tobacco Exposed Head and Neck Squamous Cell Carcinoma: A Bioinformatic Analysis. Frontiers in Oncology, 2019; 9: 1-13.

15. GORESTEIN C, ANDRADE L. Inventário de depressão de Beck : propriedades psicométricas da versão em português. Revista de Psiquiatria Clínic, 1998; 25(5): 245-250.

16. HENRY M, et al. Um algoritmo de triagem para detecção precoce de transtorno depressivo maior em pacientes com câncer de cabeça e pescoço pós tratamento: estudo longitudinal. Psicooncologia, 2018; 27(6): 1622-1628.

17. HORTENSE FTP, et al. Qualidade de vida, ansiedade e depressão de pacientes com câncer de cabeça e pescoço: estudo clínico randomizado. Revista da Escola de Enfermagem da USP, 2020; 54: 1-8.

18. HU T, et al. Relationship between resilience, social support as well as anxiety / depression of lung cancer patients: $A$ cross-sectional observation study. Journal of Cancer Research and Therapeutics, 2018; 14(1): 72.

19. HUANG C, et al. Development and validation of radiomic signatures of head and neck squamous cell carcinoma molecular features and subtypes. EBioMedicine, 2019; 45: 70-80.

20. HUANG SH, O'SULLIVAN B. Overview of the 8th Edition TNM Classification for Head and Neck Cancer. Curr Treat Options Oncol, 2017; 18(40): 2-13

21. INSTITUTO NACIONAL DO CÂNCER JOSÉ ALENCAR GOMES DA SILVA (INCA). Estimativa 2020: Incidência de câncer no Brasil. Rio de Janeiro: INCA, 2019.

22. KFOURI AS, et al. Fraction of head and neck cancer attributable to tobacco and alcohol in cities of three Brazilian regions. Revista Brasileira de Epidemiologia, 2019; 21: 1-10.

23. KIM S, et al. Pretreatment depression as a prognostic indicator of survival and nutritional status in patients with head and neck cancer. Cancer, 2016; 122 (1): 131-140.

24. LIMA RJ, et al. Resilience, functional capacity and social support of people with stroke sequelae. Revista Eletrônica de Enfermagem, 2020; 22 (59542): 1-8.

25. LEE SJ, Cartmell KB. Self - reported depression in cancer survivors versus the general population: a population - based propensity score matching- analysis. Quality Life Research, 2020; 29:483-494.

26. LUSZCZYNSKA A, et al. Self-efficacy and social support predict benefit finding 12 months after cancer surgery: The mediating role of coping strategies. Psychology, Health \& Medicine, 2005; 10(4): 365-375.

27. MELO MCB, et al. O funcionamento familiar do paciente com câncer. Psicologia em Revista, 2012; 18(1): 73-89.

28. RHOTEN BA, et al. Body image and depressive symptoms in patients with head and neck cancer: an important relationship. Support Care Cancer, 2014; 22(11): 3053-3060.

29. ROCHA BQC, et al. Características epidemiológicas de pacientes portadores de neoplasias de cabeça e pescoço submetidos à radioterapia em Juiz de Fora- MG. HU Revista, 2017; 43(1): 71-75.

30. ROHDE RL, et al. Prevalence and sociodemographic factors associated with depression among hospitalized patients with head and neck cancer- Results from a national study. Psycho-Oncology, 2018; 27: 2809-2814.

31. SAWADA NO, et al. Depression, fatigue, and health-related quality of life in head and neck cancer patients: a prospective pilot study. Support Care Cancer, 2012; 20: 2705-2711.

32. SHARPLEY CF, et al. Does resilience ' buffer' against depression in prostate cancer patients ? A multi-site replication study. European Journal of Cancer Care, 2014; 23: 545-552.

33. SIEGEL RL, et al. Cancer statistics, 2019. CA cancer journal for clinicians, 2019; 69(1): 7-34.

34. SUZUKI M, et al. Anxiety and depression in patients after surgery for head and neck cancer in Japan. Palliat Support Care, 2016; 14(3): 269-77.

35. THAMBYRAJAH C, et al. " Cancer Doesn ' t Mean Curtains ": Benefit Finding in Patients with Head and Neck Cancer in Remission. Journal of Psychosocial Oncology, 2010; 28 (6): 666-682.

36. VARTANIAN JG, et al. Brazilian-Portuguese validation of the University of Washington Quality of Life Questionnaire for patients with head and neck cancer. Head \& Neck, 2006; 28 (12): 1115- 1121.

37. WAGNILD, G. A review of the Resilience Scale. Journal of Nursing Measurement, 2009; 17(2): 105-113.

38. WAGNILD G, YOUNG H. Development and psychometric evaluation of the Resilience Scale. J Nurs Meas., 1993; 1(2): 165-78.

39. ZIMMARO LA, et al. Depressive symptoms predict head and neck cancer survival: Examining plausible behavioral and biological pathways. Cancer, 2018; 124(5): 1053-1060. 